\title{
Shilov boundary for holomorphic functions on some classical Banach spaces
}

\author{
by \\ María D. Acosta (Granada) and \\ Mary Lilian Lourenço (São Paulo)
}

\begin{abstract}
Let $\mathcal{A}_{\infty}\left(B_{X}\right)$ be the Banach space of all bounded and continuous functions on the closed unit ball $B_{X}$ of a complex Banach space $X$ and holomorphic on the open unit ball, with sup norm, and let $\mathcal{A}_{\mathrm{u}}\left(B_{X}\right)$ be the subspace of $\mathcal{A}_{\infty}\left(B_{X}\right)$ of those functions which are uniformly continuous on $B_{X}$. A subset $B \subset B_{X}$ is a boundary for $\mathcal{A}_{\infty}\left(B_{X}\right)$ if $\|f\|=\sup _{x \in B}|f(x)|$ for every $f \in \mathcal{A}_{\infty}\left(B_{X}\right)$. We prove that for $X=d(w, 1)$ (the Lorentz sequence space) and $X=C_{1}(H)$, the trace class operators, there is a minimal closed boundary for $\mathcal{A}_{\infty}\left(B_{X}\right)$. On the other hand, for $X=\mathcal{S}$, the Schreier space, and $X=K\left(\ell_{p}, \ell_{q}\right)(1 \leq p \leq q<\infty)$, there is no minimal closed boundary for the corresponding spaces of holomorphic functions.
\end{abstract}

1. Introduction. A result of Shilov asserts that if $\mathfrak{A}$ is a unital separating algebra of $\mathcal{C}(K)$ ( $K$ a compact Hausdorff topological space), then there is a smallest closed subset $S \subset K$ such that every function of $\mathfrak{A}$ attains its norm at some point of $S$ [6, Theorem I.4.2]. Bishop [4] proved that if $K$ is metrizable, then, in fact, there is a minimal subset of $K$ satisfying the above condition for every separating algebra of $\mathcal{C}(K)$. That subset is the set of peak points for $\mathfrak{A}$ (see definition below).

Globevnik introduced the corresponding concepts for a subalgebra of $\mathcal{C}_{\mathrm{b}}(\Omega)$, the space of bounded continuous functions on a topological space $\Omega$ not necessarily compact [9]. In fact, he considered the case $\Omega=B_{X}$, where $X$ is a Banach space. If $\mathfrak{A}$ is a subspace of $\mathcal{C}_{\mathrm{b}}(\Omega)$, we will say that a subset $B \subset \Omega$ is a boundary for $\mathfrak{A}$ if

$$
\|f\|=\sup _{b \in B}|f(b)|, \quad \forall f \in \mathfrak{A} .
$$

2000 Mathematics Subject Classification: Primary 46G20, 46J20; Secondary 46B99.

Key words and phrases: holomorphic function, boundary, Shilov boundary, peak point, strong peak point.

The first named author was supported in part by MEC project MTM2006-04837 and Junta de Andalucía "Proyecto de Excelencia" FQM-01438 The second named author was partially supported by CNPq (project no. 472416/2004-9). 
If there is a closed boundary $B$ that is contained in all closed boundaries for $\mathfrak{A}$, we will say that $B$ is the Shilov boundary of $\mathfrak{A}$.

If $X$ is a complex Banach space, we will denote by $\mathcal{A}_{\mathrm{u}}\left(B_{X}\right)$ the space of uniformly continuous functions on the closed unit ball of $X$ which are holomorphic on the open unit ball. Globevnik [9] described the boundaries of $\mathcal{A}_{\mathrm{u}}\left(B_{c_{0}}\right)$. As a consequence of the description, he showed that this algebra has no Shilov boundary. Aron, Choi, Lourenço and Paques [3] gave examples of boundaries for $\mathcal{A}_{\mathrm{u}}\left(B_{\ell_{\infty}}\right)$ and proved that there is no Shilov boundary for this algebra. They also showed that the unit sphere of $\ell_{1}$ is the Shilov boundary for $\mathcal{A}_{\mathrm{u}}\left(B_{\ell_{1}}\right)$.

Moraes and Romero [14] gave a characterization of the boundaries of $\mathcal{A}_{\mathrm{u}}\left(B_{d_{*}(w, 1)}\right)$, where $d_{*}(w, 1)$ is the canonical predual of the Lorentz sequence space $d(w, 1)$ when $w=(1 / n)$. Later Acosta, Moraes and Romero [2] generalized that characterization proving it for any space $d_{*}(w, 1)$ and obtained another one in terms of the strong peak sets of the unit ball. In this case, there is no Shilov boundary. Choi, García, Kim and Maestre [5] proved that there is no Shilov boundary for $\mathcal{A}_{\mathrm{u}}\left(B_{C(K)}\right)$, when $K$ is infinite and scattered. Acosta showed the same result for every infinite $K$ and also proved that for this space the set of extreme points of the unit ball of $C(K)$ is a boundary for $\mathcal{A}_{\mathrm{u}}\left(B_{C(K)}\right)$ (see $\left.[1]\right)$.

Before going on it is convenient to recall some definitions. Let $\mathcal{A}$ be a function space on a metric space $\Omega$. An element $y \in \Omega$ is called a peak point for $\mathcal{A}$ if there is some $f \in \mathcal{A}$ such that $f(y)=1$ and $|f(x)|<1$ for all $x \in \Omega \backslash\{y\}$. In this case we say that $f$ peaks at $y$. An element $y \in \Omega$ is called a strong peak point for $\mathcal{A}$ if there is some $f \in \mathcal{A}$ satisfying $f(y)=1$ and such that given any $\varepsilon>0$ there is some $\delta>0$ such that $\operatorname{dist}(x, y)>\varepsilon$ implies that $|f(x)|<1-\delta$. It is clear that every closed boundary for $\mathcal{A}$ contains all the strong peak points.

In this paper we prove that there is no Shilov boundary for $\mathcal{A}_{\mathrm{u}}\left(B_{X}\right)$ when $X$ is the Schreier space or the space $K\left(\ell_{p}, \ell_{q}\right)(1 \leq p \leq q<\infty)$. For the spaces $X=C_{1}(H)$, the trace class operators on a complex Hilbert space $H$, or $X=d(w, 1)$, the Shilov boundary for $\mathcal{A}_{\mathrm{u}}\left(B_{X}\right)$ exists. In fact, all the points in the unit sphere of $d(w, 1)$ are strong peak points for $\mathcal{A}_{\mathrm{u}}\left(B_{d(w, 1)}\right)$, and so in this case the Shilov boundary is the unit sphere. For $\ell_{1}$ the same result holds. That fact was proved in [3] for the finitely supported sequences in the unit sphere. If $K$ is infinite, we also prove that there are no strong peak points for $\mathcal{A}_{\mathrm{u}}\left(B_{C(K)}\right)$. The set of peak points for $\mathcal{A}_{\mathrm{u}}\left(B_{C(K)}\right)$ is the set of extreme points of $B_{C(K)}$ if $K$ is separable.

Throughout this paper, all the Banach spaces considered are complex. For a Banach space $X, B_{X}$ and $S_{X}$ will be the closed unit ball and the unit sphere of $X$, respectively. We will denote by $\mathcal{A}_{\infty}\left(B_{X}\right)$ the Banach space of all bounded and continuous functions on $B_{X}$ which are holomorphic on the 
open unit ball, and by $\mathcal{A}_{\mathrm{u}}\left(B_{X}\right)$ the space of all functions in $\mathcal{A}_{\infty}\left(B_{X}\right)$ which are uniformly continuous.

\section{Existence of the Shilov boundary on the Lorentz sequence} space. Given a decreasing sequence $w$ of positive real numbers satisfying $w_{1}=1$ and $w \in c_{0} \backslash \ell_{1}$, the complex Lorentz sequence space $d(w, 1)$ is given by

$$
d(w, 1)=\left\{x: \mathbb{N} \rightarrow \mathbb{C}: \sup \left\{\sum_{n=1}^{\infty}|x(\sigma(n))| w_{n}: \sigma: \mathbb{N} \rightarrow \mathbb{N} \text { injective }\right\}<\infty\right\} .
$$

The norm is given by

$$
\|x\|=\sup \left\{\sum_{n=1}^{\infty} w_{n}|x(\sigma(n))|: \sigma: \mathbb{N} \rightarrow \mathbb{N} \text { injective }\right\} \quad(x \in d(w, 1)) .
$$

It is well known and easy to verify that the above supremum is attained for the decreasing rearrangement of $x$. The usual vector basis $\left(e_{n}\right)$ is a monotone Schauder basis (see [12]).

A canonical predual $d_{*}(w, 1)$ of $d(w, 1)$ is given by

$$
d_{*}(w, 1)=\left\{x \in c_{0}: \lim _{n} \frac{\sum_{k=1}^{n} x^{*}(k)}{W_{n}}=0\right\}
$$

where $W_{n}=\sum_{k=1}^{n} w_{k}$ and $x^{*}$ is the decreasing rearrangement of $x$. This space is a Banach space endowed with the norm

$$
\|x\|=\sup _{n}\left\{\frac{\sum_{k=1}^{n} x^{*}(k)}{W_{n}}\right\}
$$

(see $[16]$ and $[7]) . d_{*}(w, 1)$ has a Schauder basis whose sequence of biorthogonal functionals is, in fact, the canonical basis of $d(w, 1)$.

We begin by presenting some useful lemmas.

Lemma 2.1. If $\left(z_{n}\right)$ is a bounded sequence of complex numbers such that the sequence $\left(1+\left|z_{n}\right|-\left|1+z_{n}\right|\right)$ converges to zero, then so does $\left(\left|z_{n}\right|-z_{n}\right)$.

Proof. We consider the following identity for a complex number $z$ :

$$
\begin{aligned}
(1+|z|-|1+z|)^{2} & =1+|z|^{2}+2|z|+|1+z|^{2}-2(1+|z|)|1+z| \\
& =2(\operatorname{Re} z-|z|)+2(1+|z|)(1+|z|-|1+z|) .
\end{aligned}
$$

If we apply the above identity to the sequence $\left(z_{n}\right)$ and use the assumption, we find that the sequence $\left(\left|z_{n}\right|-\operatorname{Re} z_{n}\right)$ converges to zero.

Now if we consider the expression

$$
\begin{aligned}
(|z|-\operatorname{Re} z)^{2} & =2(\operatorname{Re} z)^{2}+(\operatorname{Im} z)^{2}-2|z| \operatorname{Re} z \\
& =(\operatorname{Im} z)^{2}+2(\operatorname{Re} z-|z|) \operatorname{Re} z
\end{aligned}
$$


and we apply the identity to the sequence $\left(z_{n}\right)$, we deduce that $\operatorname{Im} z_{n} \rightarrow 0$. Hence

$$
\left|z_{n}\right|-z_{n}=\left|z_{n}\right|-\operatorname{Re} z_{n}-i \operatorname{Im} z_{n} \rightarrow 0 .
$$

Lemma 2.2 ([3, Lemma 9]). Let $0<a<1$. The real-valued function given by

$$
g_{a}(x)=\left(1+\frac{x}{1-a}\right)\left(1+\frac{1-x}{a}\right) \quad(x \in \mathbb{R})
$$

attains its maximum at $x=a$ and

$$
g_{a}(x)<g_{a}(a)=\frac{1}{a(1-a)}, \quad \forall x \in \mathbb{R} \backslash\{a\} .
$$

Lemma 2.3. The set of peak points in $S_{X}$ for $\mathcal{A}_{\infty}\left(B_{X}\right)$ is invariant under surjective linear isometries on $X$. The same holds for the set of strong peak points in $S_{X}$.

By the maximum modulus theorem, every peak point for a subspace of $\mathcal{A}_{\infty}\left(B_{X}\right)$ belongs to $S_{X}$. As a consequence, so does every strong peak point. The following result shows the converse for the subspace of all polynomials on $d(w, 1)$.

THEOREM 2.4. The set of strong peak points for the space of polynomials of degree less than or equal to 2 on $d(w, 1)$ contains the unit sphere of $d(w, 1)$.

Proof. Let $y_{0} \in S_{d(w, 1)}$. By Lemma 2.3 we can assume that supp $y_{0}$ is an interval of positive integers containing $\{1\}$ and

$$
y_{0}(j) \in \mathbb{R}^{+}, \quad \forall j \in \operatorname{supp} y_{0}, \quad y_{0}(n) \geq y_{0}(n+1), \quad \forall n \in \mathbb{N} .
$$

We will prove that $y_{0}$ is a strong peak point for $\mathcal{A}_{\mathbf{u}}(d(w, 1))$.

If the support of $y_{0}$ contains just one element, then $y_{0}=e_{1}$ and it is sufficient to consider the first-degree polynomial given by

$$
f(x)=1+x(1) \quad(x \in d(w, 1)) .
$$

Clearly $\|f\|=2=f\left(y_{0}\right)$. By using the fact that in $S_{d(w, 1)}$ the weak and $\sigma\left(d(w, 1), d_{*}(w, 1)\right)$ convergences coincide ([16, Proposition 2.2] and [10, Corollary III.2.15]) and that every point of the unit sphere is a point of weak-norm continuity of the unit ball [13, Proposition 4], it is easily checked that $f$ strongly peaks in the unit ball at $y_{0}$.

Now assume that $J:=\operatorname{supp} y_{0}$ has at least two elements. Since $\left\|y_{0}\right\|=1$, by (1), we know that $\sum_{i \in J} w_{i} y_{0}(i)=1$ and so $0<w_{i} y_{0}(i)<1$ for every $i \in J$.

For every $k \in J$ we define

$$
f_{k}(x)=\frac{1}{M_{k}}\left(1+\frac{w_{k} x(k)}{1-w_{k} y_{0}(k)}\right)\left(1+\frac{1}{w_{k} y_{0}(k)} \sum_{j \in J \backslash\{k\}} w_{j} x(j)\right) \quad(x \in d(w, 1)),
$$


where

$$
M_{k}=\frac{1}{w_{k} y_{0}(k)\left(1-w_{k} y_{0}(k)\right)} .
$$

Then $f_{k}$ is clearly a non-homogeneous polynomial on $d(w, 1)$ of degree 2 and $f_{k}\left(y_{0}\right)=1$. We will check that $\left\|f_{k}\right\|=1$.

If $x \in B_{d(w, 1)}$, then

$$
\begin{aligned}
\left|f_{k}(x)\right| & =\frac{1}{M_{k}}\left|1+\frac{w_{k} x(k)}{1-w_{k} y_{0}(k)}\right|\left|1+\frac{1}{w_{k} y_{0}(k)} \sum_{j \in J \backslash\{k\}} w_{j} x(j)\right| \\
& \leq \frac{1}{M_{k}}\left(1+\frac{w_{k}|x(k)|}{1-w_{k} y_{0}(k)}\right)\left(1+\frac{1}{w_{k} y_{0}(k)} \sum_{j \in J \backslash\{k\}}\left|w_{j} x(j)\right|\right) \\
& \leq \frac{1}{M_{k}}\left(1+\frac{w_{k}|x(k)|}{1-w_{k} y_{0}(k)}\right)\left(1+\frac{1-w_{k}|x(k)|}{w_{k} y_{0}(k)}\right) \quad\left(\text { since } x \in B_{X}\right) \\
& \leq \frac{1}{M_{k}}\left(1+\frac{w_{k} y_{0}(k)}{1-w_{k} y_{0}(k)}\right)\left(1+\frac{1-w_{k} y_{0}(k)}{w_{k} y_{0}(k)}\right) \quad(\text { by Lemma } 2.2) \\
& =1 .
\end{aligned}
$$

Hence $\left\|f_{k}\right\|=1$.

Our intention is to show that $y_{0}$ is a strong peak point for the space of second-degree polynomials. To this end, we will prove that

$$
x_{n} \in B_{d(w, 1)}, \forall n, \quad\left|f_{k}\left(x_{n}\right)\right| \underset{n}{\rightarrow} 1 \Rightarrow x_{n}(k) \underset{n}{\rightarrow} y_{0}(k) .
$$

For every fixed $k$, we write

$$
u_{n}=\frac{w_{k} x_{n}(k)}{1-w_{k} y_{0}(k)}, \quad v_{n}=\sum_{\substack{j \in J \\ j \neq k}} \frac{w_{j} x_{n}(j)}{w_{k} y_{0}(k)} .
$$

We rewrite the inequality (2) in terms of the above sequences:

$$
\left|f_{k}\left(x_{n}\right)\right|=\frac{1}{M_{k}}\left|1+u_{n}\right|\left|1+v_{n}\right| \leq \frac{1}{M_{k}}\left(1+\left|u_{n}\right|\right)\left(1+\left|v_{n}\right|\right) \leq 1 .
$$

If we assume that $\left|f_{k}\left(x_{n}\right)\right| \rightarrow 1$ as $n \rightarrow \infty$, then the sequence $\left(1+v_{n}\right)$ has no subsequence converging to zero. From the above inequality we deduce that

$$
\left|1+u_{n}\right|-1-\left|u_{n}\right| \rightarrow 0 \text {. }
$$

Since $k$ is fixed, Lemma 2.1 implies that $\left(\left|u_{n}\right|-u_{n}\right)$ converges to zero, that is, $\left|x_{n}(k)\right|-x_{n}(k) \rightarrow 0$ as $n \rightarrow \infty$. Also by Lemma 2.2 , we know that

$$
w_{k}\left|x_{n}(k)\right| \rightarrow w_{k} y_{0}(k) \quad \text { as } n \rightarrow \infty .
$$

Hence we deduce that $x_{n}(k) \rightarrow y_{0}(k)$ as $n \rightarrow \infty$. 
Now we choose a sequence $\left(\alpha_{n}\right)$ in $\ell_{1}$ such that $\operatorname{supp} \alpha=J, \alpha_{n}>0$ for all $n \in J$ and $\sum_{n \in J} \alpha_{n}=1$. Define

$$
f(x)=\sum_{k \in J} \alpha_{k} f_{k}(x) \quad\left(x \in B_{d(w, 1)}\right) .
$$

Then $f$ is a polynomial of degree at most 2 in $d(w, 1)$ and $\|f\| \leq 1=f\left(y_{0}\right)$.

We now prove that this function strongly peaks in the unit ball of $d(w, 1)$ at $y_{0}$. So assume that $\left|f\left(x_{n}\right)\right| \rightarrow 1$ for some sequence $\left(x_{n}\right)$ in the unit ball. Then clearly $f_{k}\left(x_{n}\right) \rightarrow 1$ as $n \rightarrow \infty$ for every $k \in J$.

Since $y_{0} \in S_{d(w, 1)}$, by condition (3), we know that $\left(x_{n}\right)$ converges pointwise to $y_{0}$. All the elements involved in the argument are in the unit ball of $d(w, 1)$ and so $\left(x_{n}\right)$ converges to $y_{0}$ in the $\sigma\left(d(w, 1), d_{*}(w, 1)\right)$-topology. Since $d_{*}(w, 1)$ is an M-ideal in its dual (see [16, Proposition 2.2] or [10, Examples III.1.4c]), in the unit ball of $d(w, 1)$, the weak and weak* topologies coincide on the unit sphere, in view of [10, Corollary III.2.15]. By applying this to the element $y_{0}$, which is the $w^{*}$-limit of $\left(x_{n}\right)$, we see that in fact $\left(x_{n}\right)$ converges weakly to $y_{0}$. Since all the points of the unit sphere of $d(w, 1)$ are points of weak-norm continuity [13, Proposition 4], we conclude that $\left(x_{n}\right)$ converges in norm to $y_{0}$ and $y_{0}$ is a strong peak point, as we wanted to show.

Corollary 2.5. The Shilov boundary for the space of second-degree polynomials on $d(w, 1)$ is $S_{d(w, 1)}$. Hence $S_{d(w, 1)}$ is also the Shilov boundary for $\mathcal{A}_{\mathrm{u}}\left(B_{d(w, 1)}\right)$ and $\mathcal{A}_{\infty}\left(B_{d(w, 1)}\right)$.

It is known that all the finitely supported elements in $S_{\ell_{1}}$ are strong peak points for the space of second-degree polynomials on $\ell_{1}[3$, Theorem 10]. We now extend that result.

THEOREM 2.6. $S_{\ell_{1}}$ is the set of strong peak points for the space of seconddegree polynomials on $\ell_{1}$.

Proof. If $y_{0} \in S_{\ell_{1}}$, then, by Lemma 2.3, we can assume that $y_{0}(n) \geq 0$ for every $n$. If $\left|\operatorname{supp} y_{0}\right|=1$ and $\{n\}=\operatorname{supp} y_{0}$, the function $x \mapsto 1+x(n)$ strongly peaks in the unit ball of $\ell_{1}$ at $y_{0}$. Otherwise, if $J:=\operatorname{supp} y_{0}$ satisfies $|J| \geq 2$, then the second-degree polynomial given by

$$
f_{k}(x):=\frac{1}{y_{0}(k)\left(1-y_{0}(k)\right)}\left(1+\frac{x(k)}{1-y_{0}(k)}\right)\left(1+\frac{\sum_{i \neq k} x(i)}{y_{0}(k)}\right) \quad\left(x \in \ell_{1}\right)
$$

satisfies $f_{k}\left(y_{0}\right)=1$. In view of Lemma 2.2 , also $\left\|f_{k}\right\|=1$ and now we can follow the argument in the proof of Theorem 2.4.

3. Boundaries for the Schreier space and $C(K)$. A subset $E=$ $\left\{n_{1}<\cdots<n_{k}\right\}$ of the natural numbers $\mathbb{N}$ is said to be admissible if $k \leq n_{1}$. The $S$ chreier space $\mathcal{S}$ is the completion of the space $c_{00}$ of all scalar sequences 
of finite support with respect to the norm $\|x\|=\sup \sum_{j \in E}\left|x_{j}\right|$, where the supremum is taken over all admissible sets $E$ of natural numbers.

The following theorem shows in particular that the intersection of all boundaries for $\mathcal{A}_{\infty}\left(B_{\mathcal{S}}\right)$ is empty.

TheOREm 3.1. Let $\mathcal{S}$ be the Schreier space and $B$ be a boundary for $\mathcal{A}_{\infty}\left(B_{\mathcal{S}}\right)$. If $x_{0} \in B$ and $0<r<1$, then $B \backslash\left(x_{0}+r B_{\mathcal{S}}\right)$ is a boundary for $\mathcal{A}_{\infty}\left(B_{\mathcal{S}}\right)$. As a consequence, there is no Shilov boundary for $\mathcal{A}_{\infty}\left(B_{\mathcal{S}}\right)$.

Proof. Assume that $h \in \mathcal{A}_{\infty}\left(B_{\mathcal{S}}\right)$. For every $0<\varepsilon<(1-r) / 2$, there is $y_{0} \in c_{00}$ such that $\left\|y_{0}\right\|<1$ and

$$
\left|h\left(y_{0}\right)\right|>\|h\|-\varepsilon .
$$

We write $k=\max \operatorname{supp} y_{0}$ and denote by $\left(P_{m}\right)$ the sequence of canonical projections associated to the usual basis of $\mathcal{S}$. Choose a positive integer $n$ such that $n>k /\left(1-\left\|y_{0}\right\|\right)$ and $\left\|\left(I-P_{n}\right)\left(x_{0}\right)\right\|<\varepsilon$. We will check that $y_{0}+\lambda y \in B_{\mathcal{S}}$ for every $\lambda \in \mathbb{C}$ with $|\lambda|=1$ and $y=\sum_{j=n+1}^{2 n}(1 / n) e_{j}$.

Let $A=E \cup F$ be an admissible set such that $E \subset\{1, \ldots, k\}$ and $\min F>k$. If $E \neq \emptyset$, then $|E|+|F| \leq k$ and

$$
\sum_{i \in E \cup F}\left|y_{0}+\lambda y(i)\right| \leq \sum_{i \in E}\left|y_{0}(i)\right|+\sum_{i \in F}|y(i)| \leq\left\|y_{0}\right\|+\frac{k}{n} \leq 1 .
$$

If $E=\emptyset$, then $\sum_{i \in F}\left|\left(y_{0}+\lambda y\right)(i)\right|=\sum_{i \in F}|y(i)| \leq 1$. So $\left\|y_{0}+\lambda y\right\| \leq 1$.

By the maximum modulus theorem, there is $\lambda_{0} \in \mathbb{C}$ with $\left|\lambda_{0}\right|=1$ such that

$$
\left|h\left(y_{0}+\lambda_{0} y\right)\right| \geq\left|h\left(y_{0}\right)\right|>\|h\|-\varepsilon .
$$

Fix $\lambda_{1} \in \mathbb{C}$ satisfying $\left|\lambda_{1}\right|=1$ and

$$
\left|h\left(y_{0}+\lambda_{0} y\right)+\lambda_{1}\right|=\left|h\left(y_{0}+\lambda_{0} y\right)\right|+1 .
$$

Since $\|y\|=1$ and $P_{n}(y)=0$, there is $y^{*} \in S_{\mathcal{S}}$ such that $y^{*}\left(\lambda_{0} y\right)=1$, $y^{*}\left(e_{j}\right)=0$ for all $j \leq n$ and so $y^{*}\left(y_{0}\right)=0$. Now, we define a holomorphic function $g$ by

$$
g(x):=h(x)+\lambda_{1} y^{*}(x) \quad\left(x \in B_{\mathcal{S}}\right) .
$$

Clearly $g \in \mathcal{A}_{\infty}\left(B_{\mathcal{S}}\right)$ and

$$
\begin{aligned}
\|h\|-\varepsilon+1 & <\left|h\left(y_{0}\right)\right|+1 \leq\left|h\left(y_{0}+\lambda_{0} y\right)\right|+y^{*}\left(\lambda_{0} y\right) \\
& =\left|g\left(y_{0}+\lambda_{0} y\right)\right| \leq\|g\| \leq\|h\|+1 .
\end{aligned}
$$

Since $B$ is a boundary there is $z_{0} \in B$ such that

$$
\left|g\left(z_{0}\right)\right|>\|h\|-\varepsilon+1 .
$$

On the other hand,

$$
\left|g\left(z_{0}\right)\right| \leq\left|h\left(z_{0}\right)\right|+\left|y^{*}\left(z_{0}\right)\right| \leq\|h\|+\left|y^{*}\left(z_{0}\right)\right| \leq\|h\|+1 .
$$


This implies $\left|y^{*}\left(z_{0}\right)\right|>1-\varepsilon$. Hence

$$
\left\|\left(I-P_{n}\right)\left(z_{0}\right)\right\| \geq\left|y^{*}\left(z_{0}\right)\right|>1-\varepsilon .
$$

Consequently,

$$
\begin{aligned}
\left\|z_{0}-x_{0}\right\| & \geq\left\|\left(I-P_{n}\right)\left(z_{0}-x_{0}\right)\right\| \\
& \geq\left\|\left(I-P_{n}\right)\left(z_{0}\right)\right\|-\left\|\left(I-P_{n}\right) x_{0}\right\| \geq 1-2 \varepsilon>r .
\end{aligned}
$$

Also $\left|h\left(z_{0}\right)\right|+1 \geq\|h\|+1-\varepsilon$ and hence $\left|h\left(z_{0}\right)\right|>\|h\|-\varepsilon$. Therefore $z_{0} \in B \backslash\left(x_{0}+r B_{\mathcal{S}}\right)$ and this set is a boundary for $\mathcal{A}_{\infty}\left(B_{\mathcal{S}}\right)$. As a consequence, the Shilov boundary of this space does not exist.

We recall that a point $x \in B_{X}$ is a $\mathbb{C}$-extreme point of the unit ball if

$$
(y \in X,\|x+\lambda y\| \leq 1, \forall \lambda \in \mathbb{C},|\lambda|=1) \Rightarrow y=0 .
$$

THEOREM 3.2. If $K$ is any infinite compact Hausdorff topological space, then there are no strong peak points for $\mathcal{A}_{\infty}\left(B_{\mathcal{C}(K)}\right)$. If $K$ is separable, then all the extreme points in $B_{\mathcal{C}(K)}$ are peak points for the space of first-degree polynomials on $\mathcal{C}(K)$.

Proof. It is known that every peak point is a $\mathbb{C}$-extreme point $[8$, Theorem 4]. So we will prove that $\mathbb{C}$-extreme points of $B_{\mathcal{C}(K)}$ are not strong peak points. Assume that $x_{0} \in S_{\mathcal{C}(K)}$ is an extreme point of the unit ball. Since $K$ is infinite, there is a sequence $\left(x_{n}\right) \subset \mathcal{C}(K)$ satisfying

$$
0 \leq x_{n} \leq 1,\left\|x_{n}\right\|=1, \forall n, \quad \operatorname{supp} x_{n} \cap \operatorname{supp} x_{m}=\emptyset, \forall n \neq m .
$$

Assume that $h \in B_{\mathcal{A}_{\infty}\left(B_{\mathcal{C}(K)}\right)}$ with $h\left(x_{0}\right)=1$. Since $\left(x_{n}\right)$ is equivalent to the $c_{0}$-basis, it converges weakly to zero. Then the sequence $\left(x_{0}\left(1-x_{n}\right)\right)$ is in the unit ball of $\mathcal{C}(K)$ and converges weakly to $x_{0}$. Since $\mathcal{C}(K)$ has the DunfordPettis property, it also has the polynomial Dunford-Pettis property [15], and so the argument in the proof of [1, Proposition 4.1] shows that

$$
h\left(x_{0}\left(1-x_{n}\right)\right) \rightarrow 1 \text {. }
$$

Since $x_{n}$ are non-negative elements in the unit sphere, for every $n$ there is $t_{n} \in K$ such that $x_{n}\left(t_{n}\right)=1$ and so

$$
\left\|x_{0}\left(1-x_{n}\right)-x_{0}\right\| \geq\left\|x_{0} x_{n}\right\| \geq\left|x_{0}\left(t_{n}\right) x_{n}\left(t_{n}\right)\right|=1 .
$$

Hence $x_{0}$ is not a strong peak point for $\mathcal{A}_{\infty}\left(B_{\mathcal{C}(K)}\right)$.

If $K$ is separable and $\left\{t_{n}: n \in \mathbb{N}\right\}$ is a dense set in $K$, we will prove that the function $u$ such that $u(K)=\{1\}$ is a peak point for the space of first-degree polynomials. In view of Lemma 2.3 , this proves the stated assertion.

Define

$$
f(x):=\sum_{n=1}^{\infty} \alpha_{n}\left(1+x\left(t_{n}\right)\right) \quad(x \in \mathcal{C}(K)),
$$


where $\left(\alpha_{n}\right) \subset S_{\ell_{1}}$ with $\alpha_{n}>0$ for every $n$. Then $f$ is clearly a first-degree polynomial on $\mathcal{C}(K)$ and $f(u)=\|f\|=2$. If $x \in B_{\mathcal{C}(K)}$ and $|f(x)|=2$, then $\left|1+x\left(t_{n}\right)\right|=2$ for every $n$ and so $x\left(t_{n}\right)=1$ for all $n$, that is, $x=u$.

Since $\ell_{\infty}$ has a countable subset of functionals that separate points and attain the norm at the same element of the unit ball, we can also obtain:

Corollary $3.3([3])$. All the extreme points in $B_{\ell_{\infty}}$ are peak points for the space of first-degree polynomials on $\ell_{\infty}$.

4. Shilov boundary on the trace class operators. Let $H$ be a complex Hilbert space. An operator $T: H \rightarrow H$ is called a trace class operator if there are orthonormal sequences $\left(e_{n}\right)$ and $\left(f_{n}\right)$ in $H$ such that $T(x)=\sum_{n=1}^{\infty} \lambda_{n}\left\langle x, e_{n}\right\rangle f_{n}$ for every $x \in H$ and the sequence $\left(\lambda_{n}\right)$ is in $\ell_{1}$. In that case, the norm of $T$ is given by $\|T\|=\sum_{n=1}^{\infty}\left|\lambda_{n}\right|$. We denote by $C_{1}(H)$ the Banach space of all trace class operators on $H$.

TheOREm 4.1. If $H$ is a complex Hilbert space, then the Shilov boundaries for $\mathcal{A}_{\mathrm{u}}\left(C_{1}(H)\right)$ and $\mathcal{A}_{\infty}\left(C_{1}(H)\right)$ both exist and coincide.

Proof. Assume that $\left\{e_{i}: i \in I\right\}$ is an orthonormal basis of $H$ and $F \subset I$ is any subset. Then the operator $\Pi_{F}$ given by

$$
\Pi_{F}(T):=P_{F} T P_{F} \quad\left(T \in C_{1}(H)\right),
$$

where $P_{F}(x)=\sum_{i \in F} x(i) e_{i}(x \in H)$, is a norm one projection on $C_{1}(H)$. Since $\operatorname{Lin}\left\{e_{i} \otimes e_{j}: i, j \in I\right\}$ is dense in $C_{1}(H)$, for every $h \in \mathcal{A}_{\infty}\left(B_{C_{1}(H)}\right)$ we have

$$
\|h\|=\sup _{\substack{F \subset I \\ F \text { finite }}}\left\|h \circ \Pi_{F}\right\| .
$$

For every complex finite-dimensional space $Y$, the subset of peak points of $B_{Y}$ is a boundary for $\mathcal{A}_{\mathrm{u}}\left(B_{Y}\right)$ [4, Theorem 1]. We will prove that for every finite subset $F \subset I$, every peak point of the unit ball of $\Pi_{F}\left(C_{1}(H)\right)$ for the space of bounded and continuous functions on the unit ball of $\Pi_{F}\left(C_{1}(H)\right)$ which are holomorphic on the open unit ball, is a strong peak point for $\mathcal{A}_{\mathrm{u}}\left(B_{C_{1}(H)}\right)$.

Let $T_{0} \in S_{C_{1}(H)} \cap \Pi_{F}\left(C_{1}(H)\right)$ be a peak point. Then there is a continuous function $g$ on the unit ball of $\Pi_{F}\left(C_{1}(H)\right)$, which is holomorphic on the open unit ball and satisfies

$$
g\left(T_{0}\right)=\|g\|=1 \quad \text { and } \quad|g(T)|<1, \forall T \in\left(B_{C_{1}(H)} \cap \Pi_{F}\left(C_{1}(H)\right)\right) \backslash\left\{T_{0}\right\} .
$$

Now we extend $g$ to $B_{C_{1}(H)}$ by

$$
\widetilde{g}(T)=g\left(\Pi_{F}(T)\right) \quad\left(T \in B_{C_{1}(H)}\right) .
$$

Clearly $\widetilde{g} \in \mathcal{A}_{\mathrm{u}}\left(B_{C_{1}(H)}\right),\|\widetilde{g}\| \leq\|g\|=1$ and $\widetilde{g}\left(T_{0}\right)=1$. Assume that $\left(T_{n}\right) \subset B_{C_{1}(H)}$ with $\left|\widetilde{g}\left(T_{n}\right)\right| \rightarrow 1$, that is, $\left|g\left(\Pi_{F}\left(T_{n}\right)\right)\right| \rightarrow 1$. Since $\Pi_{F}\left(C_{1}(H)\right)$ 
is a finite-dimensional space and $T_{0}$ is a peak point, we have $\Pi_{F}\left(T_{n}\right) \rightarrow T_{0}$. Since $\left\|T_{0}\right\|=1$, it follows that $\left\|\Pi_{F}\left(T_{n}\right)\right\| \rightarrow 1$. By using [11, Proposition 2.2], we have

$$
\begin{array}{r}
\left\|P_{F} T_{n} P_{F}\right\|^{2}+\left\|P_{F} T_{n}\left(I-P_{F}\right)\right\|^{2}+\left\|\left(I-P_{F}\right) T_{n} P_{F}\right\|^{2}+\left\|\left(I-P_{F}\right) T_{n}\left(I-P_{F}\right)\right\|^{2} \\
\leq\left\|T_{n}\right\|^{2} \leq 1,
\end{array}
$$

and so $\left\|\Pi_{F}\left(T_{n}\right)-T_{n}\right\|=\left\|P_{F} T_{n} P_{F}-T_{n}\right\| \rightarrow 0$. Since we know that $\left(\Pi_{F}\left(T_{n}\right)\right)$ converges to $T_{0}$, so does $\left(T_{n}\right)$, and $T_{0}$ is a strong peak point, as we wanted to show. Since the strong peak points are contained in any closed boundary and in this case the set of strong peak points is a boundary for $\mathcal{A}_{\mathrm{u}}\left(B_{C_{1}(H)}\right)$, the Shilov boundary for this space is the closure of the set of strong peak points of $\mathcal{A}_{\mathrm{u}}\left(B_{C_{1}(H)}\right)$. The same argument works for $\mathcal{A}_{\infty}\left(B_{C_{1}(H)}\right)$.

5. Boundaries for $K\left(\ell_{p}, \ell_{q}\right)$. We now study the properties of the boundaries for $\mathcal{A}_{\infty}\left(B_{X}\right)$, where $X$ is the space of all compact operators on $\ell_{p}$ for $1 \leq p<\infty$.

TheOREm 5.1. If $1 \leq p \leq q<\infty$, then there is no Shilov boundary for $\mathcal{A}_{\infty}\left(B_{K\left(\ell_{p}, \ell_{q}\right)}\right)$. In fact, if $B$ is a boundary for $\mathcal{A}_{\infty}\left(B_{K\left(\ell_{p}, \ell_{q}\right)}\right), 0<r<1$ and $S_{0} \in B$, then $B \backslash\left(S_{0}+r B_{K\left(\ell_{p}, \ell_{q}\right)}\right)$ is also a boundary for $\mathcal{A}_{\infty}\left(B_{K\left(\ell_{p}, \ell_{q}\right)}\right)$. There are closed boundaries $A, B$ for $\mathcal{A}_{\infty}\left(B_{K\left(\ell_{p}, \ell_{q}\right)}\right)$ such that $\operatorname{dist}(A, B) \geq 1$. The same assertions hold for $\mathcal{A}_{\mathrm{u}}\left(B_{K\left(\ell_{p}, \ell_{q}\right)}\right)$.

Proof. We denote by $\left(P_{n}\right)$ and $\left(Q_{n}\right)$ the sequences of canonical projections associated to the usual bases of $\ell_{p}$ and $\ell_{q}$, respectively.

Assume that $B \subset B_{K\left(\ell_{p}, \ell_{q}\right)}$ is a boundary for $\mathcal{A}_{\infty}\left(B_{K\left(\ell_{p}, \ell_{q}\right)}\right), 0<r<1$ and $S_{0} \in B$. If $h \in \mathcal{A}_{\infty}\left(B_{K\left(\ell_{p}, \ell_{q}\right)}\right)$ and $0<\varepsilon<(1-r) / 3$, then there are $N \in \mathbb{N}$ and $F \in B_{K\left(\ell_{p}, \ell_{q}\right)}$ which satisfy $Q_{N} F P_{N}=F$ and

$$
|h(F)|>\|h\|-\varepsilon \text {. }
$$

Since $S_{0}$ is a compact operator, there exists $n>N$ with

$$
\left\|\left(I-Q_{n}\right) S_{0}\left(I-P_{n}\right)\right\|<\varepsilon .
$$

Choose $R \in S_{K\left(\ell_{p}, \ell_{q}\right)}$ such that

$$
\left(I-Q_{n}\right) R\left(I-P_{n}\right)=R,
$$

and $x_{0} \in S_{\ell_{p}}$ satisfying $P_{n} x_{0}=0$ and $\left\|R\left(x_{0}\right)\right\|=1$. Then there exists $y^{*} \in S_{\ell_{q}^{*}}$ with $Q_{n}^{*}\left(y^{*}\right)=0$ and $y^{*}\left(R\left(x_{0}\right)\right)=1$. Notice that $\|F+\lambda R\| \leq 1$ for every complex number $\lambda$ with $|\lambda|=1$. By the maximum modulus theorem, there is $\lambda_{0} \in \mathbb{C}$ such that $\left|\lambda_{0}\right|=1$ and

$$
|h(F)| \leq\left|h\left(F+\lambda_{0} R\right)\right| \leq \sup _{|\lambda|=1}|h(F+\lambda R)| .
$$

If $\lambda_{1} \in \mathbb{C}$ is a modulus one scalar satisfying

$$
\left|h\left(F+\lambda_{0} R\right)+\lambda_{1} y^{*}\left(\lambda_{0} R\left(x_{0}\right)\right)\right|=\left|h\left(F+\lambda_{0} R\right)\right|+1,
$$


we define a holomorphic function $g$ by

$$
g(T):=h(T)+\lambda_{1} y^{*}\left(T x_{0}\right) \quad\left(T \in B_{K\left(\ell_{p}, \ell_{q}\right)}\right) .
$$

Clearly $g \in \mathcal{A}_{\infty}\left(B_{K\left(\ell_{p}, \ell_{q}\right)}\right)$ and

$$
\begin{aligned}
\|g\| & \geq\left|g\left(F+\lambda_{0} R\right)\right|=\left|h\left(F+\lambda_{0} R\right)+\lambda_{1} y^{*}\left(\lambda_{0} R x_{0}\right)\right| \\
& =\left|h\left(F+\lambda_{0} R\right)\right|+1 \geq|h(F)|+\left|y^{*}\left(R x_{0}\right)\right|>\|h\|-\varepsilon+1 .
\end{aligned}
$$

Since $B$ is a boundary for $\mathcal{A}_{\infty}\left(B_{K\left(\ell_{p}, \ell_{q}\right)}\right)$, there is $S \in B$ such that $|g(S)|>$ $\|g\|-\varepsilon$. Hence

$$
\|h\|-2 \varepsilon+1 \leq\|g\|-\varepsilon<|g(S)| \leq|h(S)|+\left|y^{*}\left(S x_{0}\right)\right|,
$$

and so

$$
\left|y^{*}\left(S x_{0}\right)\right| \geq 1-2 \varepsilon
$$

By the choice of $x_{0}$ and $y^{*}$,

$$
\left\|\left(I-Q_{n}\right) S\left(I-P_{n}\right)\right\| \geq\left|y^{*}\left(I-Q_{n}\right) S\left(I-P_{n}\right) x_{0}\right|=\left|y^{*}\left(S x_{0}\right)\right| \geq 1-2 \varepsilon .
$$

Finally, we deduce that

$$
\begin{aligned}
\left\|S-S_{0}\right\| & \geq\left\|\left(I-Q_{n}\right)\left(S-S_{0}\right)\left(I-P_{n}\right)\right\| \\
& \geq\left\|\left(I-Q_{n}\right) S\left(I-P_{n}\right)\right\|-\left\|\left(I-Q_{n}\right) S_{0}\left(I-P_{n}\right)\right\| \geq 1-3 \varepsilon>r .
\end{aligned}
$$

From inequality (4), we also obtain

$$
|h(S)| \geq\|h\|-2 \varepsilon \text {. }
$$

We have just proved that $B \backslash\left(S_{0}+r B_{K\left(\ell_{p}, \ell_{q}\right)}\right)$ is a boundary for $\mathcal{A}_{\infty}\left(B_{K\left(\ell_{p}, \ell_{q}\right)}\right)$. As a consequence, the Shilov boundary of this space does not exist.

Now we give a procedure to construct boundaries for $\mathcal{A}_{\infty}\left(B_{K\left(\ell_{p}, \ell_{q}\right.}\right)$. Since $\operatorname{Lin}\left\{x \otimes y: x \in\left(\ell_{p}\right)^{*}, y \in \ell_{q}, \operatorname{supp} x, \operatorname{supp} y\right.$ are finite $\}$ is dense in $K\left(\ell_{p}, \ell_{q}\right)$, for every $h \in \mathcal{A}_{\infty}\left(B_{K\left(\ell_{p}, \ell_{q}\right)}\right)$ we have

$$
\|h\|=\sup \left\{\left\|h_{F}\right\|: F \subset \mathbb{N} \text { finite }\right\}
$$

where $h_{F}(T):=h\left(Q_{F} T P_{F}\right)$ for $T \in K\left(\ell_{p}, \ell_{q}\right)$ and $P_{F}, Q_{F}$ are the projections given by

$$
P_{F}(x)=\sum_{n \in F} x(n) e_{n} \quad\left(x \in \ell_{p}\right), \quad Q_{F}(x)=\sum_{n \in F} x(n) e_{n} \quad\left(x \in \ell_{q}\right) .
$$

Note also that $\left\|h_{F}\right\| \leq\left\|h_{G}\right\|$ for $F \subset G$.

Assume that $\left(F_{n}\right)$ is an increasing sequence of finite subsets of $\mathbb{N}$ such that $G_{n}:=F_{n+1} \backslash F_{n}$ is non-empty and $\bigcup_{n} F_{n}=\mathbb{N}$. We consider the subsets $A_{n}$ whose elements are those operators $T \in B_{K\left(\ell_{p}, \ell_{q}\right)}$ that admit a decomposition $T=R+S$ satisfying

$$
\|R\|=\|S\|=1, \quad R=Q_{F_{n}} R P_{F_{n}}, \quad Q_{F_{n}} S P_{F_{n}}=0, \quad Q_{G_{n}} S P_{G_{n}}=S .
$$

Note that $A_{n}$ is closed for every $n$. 
We now check that $B=\bigcup_{n} A_{n}$ is a closed boundary for $\mathcal{A}_{\infty}\left(B_{K\left(\ell_{p}, \ell_{q}\right)}\right)$. Given $h \in \mathcal{A}_{\infty}\left(B_{K\left(\ell_{p}, \ell_{q}\right)}\right)$ and $\varepsilon>0$, there is some finite subset $F \subset \mathbb{N}$ such that $\left\|h_{F}\right\|>\|h\|-\varepsilon$. If $F \subset F_{m}$, then also $\left\|h_{F_{m}}\right\| \geq\|h\|-\varepsilon$. Hence there is an operator $R \in S_{K\left(\ell_{p}, \ell_{q}\right)}$ such that $Q_{F_{m}} R P_{F_{m}}=R$ where $h_{F_{m}}$ attains its norm and so

$$
|h(R)| \geq\|h\|-\varepsilon \text {. }
$$

If $S \in S_{K\left(\ell_{p}, \ell_{q}\right)}$ satisfies $Q_{F_{m}} S P_{F_{m}}=0$ and $Q_{G_{m}} S P_{G_{m}}=S$, then the operator $R+\lambda S$ is in the unit ball of $K\left(\ell_{p}, \ell_{q}\right)$, for every complex number $\lambda$ in the unit disk. The maximum modulus theorem applied to the function $\lambda \mapsto h(R+\lambda S)$ defined on the complex unit disk shows that there is a complex number $\lambda_{0}$ with $\left|\lambda_{0}\right|=1$ and such that

$$
\left|h\left(R+\lambda_{0} S\right)\right| \geq|h(R)| \geq\|h\|-\varepsilon .
$$

Since $R+\lambda_{0} S \in A_{m}, B$ is a boundary for $\mathcal{A}_{\infty}\left(B_{K\left(\ell_{p}, \ell_{q}\right)}\right)$.

Note that for two positive integers $n<m$, if $T_{n} \in A_{n}$ and $T_{m} \in A_{m}$, then

$$
\left\|T_{m}-T_{n}\right\| \geq\left\|Q_{G_{m}}\left(T_{m}-T_{n}\right) P_{G_{m}}\right\|=\left\|Q_{G_{m}} T_{m} P_{G_{m}}\right\|=1 .
$$

Since every $A_{n}$ is closed, the above inequality shows that $B$ is also closed.

By the same argument, $\bigcup_{n} A_{2 n}$ and $\bigcup_{n} A_{2 n-1}$ are also closed boundaries for $\mathcal{A}_{\infty}\left(B_{K\left(\ell_{p}, \ell_{q}\right)}\right)$. In view of (5), the distance between them is at least 1 .

Acknowledgements. This note was prepared while the first named author visited the Universidade de São Paulo and the second one visited the Universidad de Granada. The authors thank both institutions for the support received and the hospitality. They are also grateful to the referee for his/her careful reading.

\section{References}

[1] M. D. Acosta, Boundaries for spaces of holomorphic functions on $\mathcal{C}(K)$, Publ. Res. Inst. Math. Sci. 42 (2006), 27-44.

[2] M. D. Acosta, L. A. Moraes and L. Romero Grados, On boundaries on the predual of the Lorentz sequence space, preprint.

[3] R. M. Aron, Y. S. Choi, M. L. Lourenço and O. W. Paques, Boundaries for algebras of analytic functions on infinite dimensional Banach spaces, in: Banach Spaces, B. L. Lin and W. B. Johnson (eds.), Contemp. Math. 144, Amer. Math. Soc., 1993, $15-22$.

[4] E. Bishop, A minimal boundary for function algebras, Pacific J. Math. 9 (1959), 629-642.

[5] Y. S. Choi, D. García, S. G. Kim and M. Maestre, Norm or numerical radius attaining polynomials on $C(K)$, J. Math. Anal. Appl. 295 (2004), 80-96.

[6] T. W. Gamelin, Uniform Algebras, Chelsea, New York, 1984.

[7] D. J. H. Garling, On symmetric sequence spaces, Proc. London Math. Soc. 16 (1966), 85-106. 
[8] J. Globevnik, On interpolation by analytic maps in infinite dimensions, Math. Proc. Cambridge Philos. Soc. 83 (1978), 243-252.

[9] —, Boundaries for polydisc algebras in infinite dimensions, ibid. 85 (1979), 291-303.

[10] P. Harmand, D. Werner and W. Werner, M-Ideals in Banach Spaces and Banach Algebras, Lecture Notes in Math. 1547, Springer, Berlin, 1993.

[11] C. Lennard, $\mathcal{C}_{1}$ is uniformly Kadec-Klee, Proc. Amer. Math. Soc. 109 (1990), 71-77.

[12] J. Lindenstrauss and L. Tzafriri, Classical Banach Spaces I, II, Springer Monographs in Math., Springer, London, 1991.

[13] A. Moltó, V. Montesinos and S. Troyanski, On quasi-denting points, denting faces and the geometry of the unit ball of $d(w, 1)$, Arch. Math. (Basel) 63 (1994), 45-55.

[14] L. A. Moraes and L. Romero Grados, Boundaries for algebras of holomorphic functions, J. Math. Anal. Appl. 281 (2003), 575-586.

[15] R. A. Ryan, Dunford-Pettis properties, Bull. Acad. Polon. Sci. Sér. Sci. Math. 27 (1979), 373-379.

[16] D. Werner, New classes of Banach spaces which are M-ideals in their biduals, Math. Proc. Cambridge Philos. Soc. 111 (1992), 337-354.

Departamento de Análisis Matemático

Facultad de Ciencias

Universidad de Granada

18071 Granada, Spain

E-mail: dacosta@ugr.es
Departamento de Matemática e Estatística Universidade de São Paulo CP 66281 CEP

05311-970 São Paulo, Brazil E-mail: mllouren@ime.usp.br

Received December 15, 2005

Revised version November 19, 2006 